\title{
Severe Rhabdomyolysis during Treatment with Trabectedin in Combination with a Herbal Drug in a Patient with Metastatic Synovial Sarcoma: A Case Report
}

\author{
Angela Damato ${ }^{a} \quad$ Mario Larocca ${ }^{a}$ Ermanno Rondini ${ }^{a} \quad$ Massimo Menga $^{b}$ \\ Carmine Pinto $^{a} \quad$ Annibale Versari $^{b}$ \\ ${ }^{a}$ Medical Oncology Unit, Clinical Cancer Centre, IRCCS Arcispedale S. Maria Nuova, \\ Reggio Emilia, Italy; ${ }^{b}$ Nuclear Medicine Unit, IRCCS Arcispedale S. Maria Nuova, \\ Reggio Emilia, Italy
}

\section{Keywords}

Rhabdomyolysis · Sarcoma $\cdot$ Trabectedin $\cdot$ Herbal drug

\begin{abstract}
Rhabdomyolysis is defined as dissolution of striped muscle characterized by leakage of intracellular muscle components into the circulation, which can ultimately lead to renal failure with a possible fatal outcome. This is an uncommon side effect of trabectedin which is used in second-line therapy of metastatic sarcoma after anthracycline and ifosfamide failure. Here, we describe a case of reversible rhabdomyolysis in a male patient with recurrent metastatic synovial sarcoma of the hand, with marked ${ }^{18} \mathrm{~F}$-FDG uptake into his skeletal muscles, after 4 cycles of trabectedin, and who at the same time was taking an alternative medicine (bioflavonoids) suspected of triggering this adverse event.

(C) 2017 The Author(s)

Published by S. Karger AG, Basel
\end{abstract}




\section{Case Reports in Oncology}

Case Rep Oncol 2017;10:258-264
\begin{tabular}{l|l}
\hline DOI: $10.1159 / 000464440$ & $\begin{array}{l}\text { C } 2017 \text { The Author(s). Published by S. Karger AG, Basel } \\
\text { www.karger.com/cro }\end{array}$
\end{tabular}

Damato et al.: Severe Rhabdomyolysis during Treatment with Trabectedin in

Combination with a Herbal Drug in a Patient with Metastatic Synovial Sarcoma

\section{Case Presentation}

This is the case of a 50-year-old Caucasian man from Italy, nonsmoker, with no alcohol or illicit drug intake. His oncology history began in July 2007 in another cancer center, after the surgical removal of an expansive lesion on his left hand achieving a complete negative margin (R0) resection. Histological findings of the lesion highlighted a monophasic synovial sarcoma. He was administered adjuvant local radiotherapy and was then followed up. In June 2008, a computed tomography (CT) scan detected local recurrence of the disease and appearance of new nodules in the middle and apical right pulmonary lobe; therefore, the patient underwent another surgery of the hand and local excision of the lung tumor, which was confirmed by the previous histological diagnosis. Taking into account the dissemination of the disease, in August 2008, he was treated with systemic chemotherapy including 4 cycles of epirubicin in combination with ifosfamide. After about 1 year, in May 2009, progression of the disease into the right lung was recorded; therefore, the patient underwent another 3 wedge resections (anterior segment of the lower and upper right lobe), which is a diagnostic for metastasis of synovial sarcoma. He was followed up with only clinical and instrumental controls (CT scan and laboratory tests). After several years, in June 2012, a contralateral progression of the pulmonary disease was detected (1 lesion in the lower left lobe) and CT scan showed pathological mediastinal lymph nodes. A wedge resection of the dorsal segment of the lower lobe was performed, histologically compatible with metastases, and 2 hilar and lobar lymph nodes, which had become inflammatory, were removed. According to the patient, he continued to be followed up until December 2015 when he was submitted once again to a wedge resection of the middle lobe for sarcoma metastases. After 10 months, the patient reported intermittent episodes of hemoptysis in the absence of other symptoms; the CT scan showed significant thoracic disease progression associated with pulmonary artery compression. Therefore, in October 2016, he started chemotherapy with trabectedin at a dose of $1.5 \mathrm{mg} / \mathrm{m}^{2}$, administered as a 24-h continuous intravenous infusion repeated every 21 days. The treatment was well tolerated for the first 3 cycles in the absence of toxicity. He continued with 4 cycles of trabectedin in December of last year. After 2 weeks, the patient began to complain of sudden weakness, difficulty walking and diffuse muscle pain until it became unbearable. The patient was referred to the emergency department of our hospital and then admitted to start symptomatic therapy and supportive care. The ECOG performance status was grade 3 . The laboratory test was found to mark an increase in serum levels of myoglobin, creatine phosphokinase (CPK), and liver function (Table 1). No kidney function injury was present. Fluorine-18 fluorodeoxyglucose ( $\left.{ }^{18} \mathrm{~F}-\mathrm{FDG}\right)$ positron emission tomography was performed and showed an intense widespread tracer uptake in the skeletal muscles, particularly in the upper extremities, sternocleidomastoid muscle, the long muscles of the neck, the muscle groups of the scapular tracks bilaterally, certain muscles of the chest wall, the rectus abdominis muscles, the iliopsoas muscles, and almost all the muscle groups of the upper and lower limbs (Fig. 1), according to the clinical history and laboratory data. When asking the patient about his medical history, he did not report any muscle trauma but he had been taking a herbal drug for hemorrhoids (diosmin) during the last course of trabectedin. After hospitalization and stopping the intake of diosmin with parenteral hydration, a complete normalization of the laboratory findings was obtained (Table 1) associated with a progressive recovery of muscle strength and mobilization of the patient starting from day 


\section{Case Reports in Oncology}

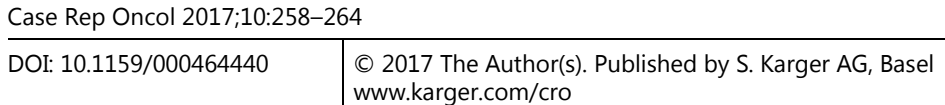
www.karger.com/cro

Damato et al.: Severe Rhabdomyolysis during Treatment with Trabectedin in Combination with a Herbal Drug in a Patient with Metastatic Synovial Sarcoma

10 with a walking aid until complete full functional recovery and a recovery of ECOG performance status grade 0 by day 15 .

\section{Discussion}

Trabectedin is an effective drug in patients with advanced sarcoma who previously failed standard of care chemotherapy [1]. It has a manageable safety profile with common toxicities including reversible low absolute neutrophil count, anemia, thrombocytopenia, and hepatotoxicity and rare, severe clinical consequences such as elevated serum CPK and rhabdomyolysis. Its etiology is unclear and is an uncommon side effect of trabectedin with an incidence of $0.5-0.7 \%[2,3]$; during preclinical studies, only sporadically increased CPK was found. Rhabdomyolysis is an injury of skeletal muscle that releases potentially toxic muscle cell components (e.g., myoglobin, other intracellular proteins, and electrolytes) into the extracellular fluid and blood stream, which may result in renal damage $[1,4,5]$. Rhabdomyolysis may have a number of causes, including direct trauma, excessive muscular activity, body temperature extremes, muscle hypoxia, infections, metabolic and electrolyte disorders, endocrine disorders, connective tissue disorders, drugs, and toxins. Overall, the clinical features of rhabdomyolysis are quite variable and they can be summarized as muscular signs and symptoms (pain, weakness, tenderness, and contractures) and other symptoms (illness, fatigue, fever, tachycardia, nausea, and vomiting). Rhabdomyolysis can result in lifethreatening renal and multiorgan failure with hypovolemia, hyperkalemia, metabolic acidosis, disseminated intravascular coagulation, and compartment syndrome, secondary arrhythmias, or cardiac arrest [3,5-7]. Biochemically, rhabdomyolysis is defined by marked blood CPK elevation (typically greater than 10 times the upper limit of normal) with creatinine increase $[8,9]$. CPK elevation is the most sensitive marker for skeletal muscle injury [10]. Trabectedin is eliminated through hepatic metabolism and CYP3A4 is the principal responsible enzyme. As a high number of drugs are also metabolized by this enzyme, interactions with multiple concomitant drugs could arise. According to in vitro data, trabectedin exposure could be increased in the presence of CYP3A4 inhibitors [11]. Therefore, coadministration of trabectedin with potent CYP34A inhibitors should be avoided or, if this is not possible, closer patient monitoring is required. The literature described a case of an association of trabectedin with a "natural foe" such as chokeberry (Aronia melanocarpa) extract derived from the black fruit of this shrub [12], which has a long tradition in European and North American folk medicine [13]. It is a relatively concentrated source of flavonoids such as quercetin, and is also reported to strongly inhibit CYP3A4 activity in the liver. It is capable of increasing the bioavailability of different chemotherapeutic agents [14]. Technical data are not listed among the flavonoid inhibitors of CYP3A4. There are several causes of exaggerated physiological ${ }^{18} \mathrm{~F}-\mathrm{FDG}$ uptake. It may occur if there is activity before, during, or after the injection of the tracer; however, this typically involves the entire muscle more or less uniformly, unlike the patchy appearance in this case. Furthermore, our patient could not move. There may also be preferential uptake of ${ }^{18} \mathrm{~F}-\mathrm{FDG}$ into muscles in hyperinsulinemic states, for example after the use of corticosteroids. The present patient had a normal glucose level at the time of tracer injection and was not taking any medications that might have affected his glucose metabolism. In the literature, several reports of tracer uptake in muscles 
have been described, e.g., in a patient taking some drugs such as tacrolimus, which was hypothesized to be due to altered glucose metabolism [15] and in a patient with statin-induced rhabdomyolysis [16]. In conclusion, in our case, according to Strippoli et al. [12], it is possible to assume a role of drug-drug interactions in the pathogenesis of this rare trabectedin side effect. Furthermore, in the presence of unexpected toxicity during conventional anticancer treatment, the simultaneous use by the patient of alternative drugs should be suspected. Although this is a rare side effect of herbal drug use, these medications are in widespread use and it is very important to remember the drug interactions and in particular inhibition of the CYP3A4 isoenzyme by several medications. It is clinically important that there is prevention and early recognition and accurate diagnosis of rhabdomyolysis. This condition may become apparent with abnormal imaging findings and the radiologist should be aware of this possible mode of presentation, which is useful for making a definitive diagnosis.

\section{Statement of Ethics}

The authors have no ethical conflicts to disclose.

\section{Disclosure Statement}

The authors have no conflicts of interest to declare.

\section{References}

1 Demetri GD, Chawla SP, von Mehren M, Ritch P, Baker LH, Blay JY, Hande KR, Keohan ML, Samuels BL, Schuetze S, Lebedinsky C, Elsayed YA, Izquierdo MA, Gomez J, Park YC, Le Cesne A: Efficacy and safety of trabectedin in patients with advanced or metastatic liposarcoma or leiomyosarcoma after failure of prior anthracyclines and ifosfamide: results of a randomized phase II study of two different schedules. J Clin Oncol 2009;27:4188-4196.

2 Grosso F, D'Incalci M: Problems in dealing with very rare adverse effects of new anticancer drugs: the example of trabectedin. Tumori 2011;97:256.

Khan FY: Rhabdomyolysis: a review of the literature. Neth J Med 2009;67:272-283.

Singh D, Chander V, Chopra K: Rhabdomyolysis. Methods Find Exp Clin Pharmacol 2005;27:39-48. Bosh X, Poch E, Grau JM: Rhabdomyolysis and acute kidney injury. N Eng J Med 2012;361:62-72.

Chatzizisis YS, Misirli G, Hatzitolios AI, Giannoglou GD: The syndrome of rhabdomyolysis: complications and treatment. Eur J Intern Med 2008;19:568-574.

7 Grosso F, D'Incalci M, Cartoafa M, Nieto A, Fernandez-Teruel C, Alfaro V, Lardelli P, Roy E, Gomez J, Kahatt C, Soto-Matos A, Judson I: A comprehensive safety analysis confirms rhabdomyolysis as an uncommon adverse reaction in patients treated with trabectedin. Cancer Chemother Pharmacol 2012;69:1557-1565.

-8 Cziraky MJ, Willey VJ, McKenney JM, Kamat SA, Fisher MD, Guyton JR, Jacobson TA, Davidson MH: Statin safety: an assessment using an administrative claims database. Am J Cardiol 2006;97:61C-68C.

-9 Pasternak RC, Smith SC Jr, Bairey-Merz CN, Grundy SM, Cleeman JI, Lenfant C: ACC/AHA/NHLBI clinical advisory on the use and safety of statins. Circulation 2002;106:1024-1028.

10 Cervellin G, Comelli I, Lippi G: Rhabdomyolysis: historical background, clinical, diagnostic and therapeutic features. Clin Chem Lab Med 2010;48:749-756. 


\section{Case Reports in Oncology}

11 Brandon EF, Sparidans RW, Guijt KJ, Lowenthal S, Meijerman I, Beijnen JH, Schellens JH: In vitro characterization of the human biotransformation and CYP reaction phenotype of ET-743 (Yondelis, Trabectidin), a novel marine anti-cancer drug. Invest New Drugs 2006;24:3-14.

$\$ 12$ Strippoli S, Lorusso V, Albano A, Guida M: Herbal-drug interaction induced rhabdomyolysis in a liposarcoma patient receiving trabectedin. BMC Complement Altern Med 2013;13:199.

13 Kulling SE, Rawel HM: Chokeberry (Aronia melanocarpa) - a review on the characteristic components and potential health effects. Planta Med 2008;74:1625-1634

14 Choi JS, Piao YJ, Kang KW: Effects of quercetin on the bioavailability of doxorubicin in rats: role of CYP3A4 and P-gp inhibition by quercetin. Arch Pharm Res 2011;34:607- 613.

15 Groves AM, Cheow HK, Win T, et al: Extensive skeletal muscle uptake of ${ }^{18} \mathrm{~F}$-FDG: relation to immunosuppressants? J Nucl Med Technol 2004;32:206e8.

16 Sheehy N, Israel DA: Findings on ${ }^{18}$ FDG-PET imaging in statin-induced rhabdomyolysis. Clin Radiol 2007;62:1012-1014. 


\section{Case Reports in Oncology}
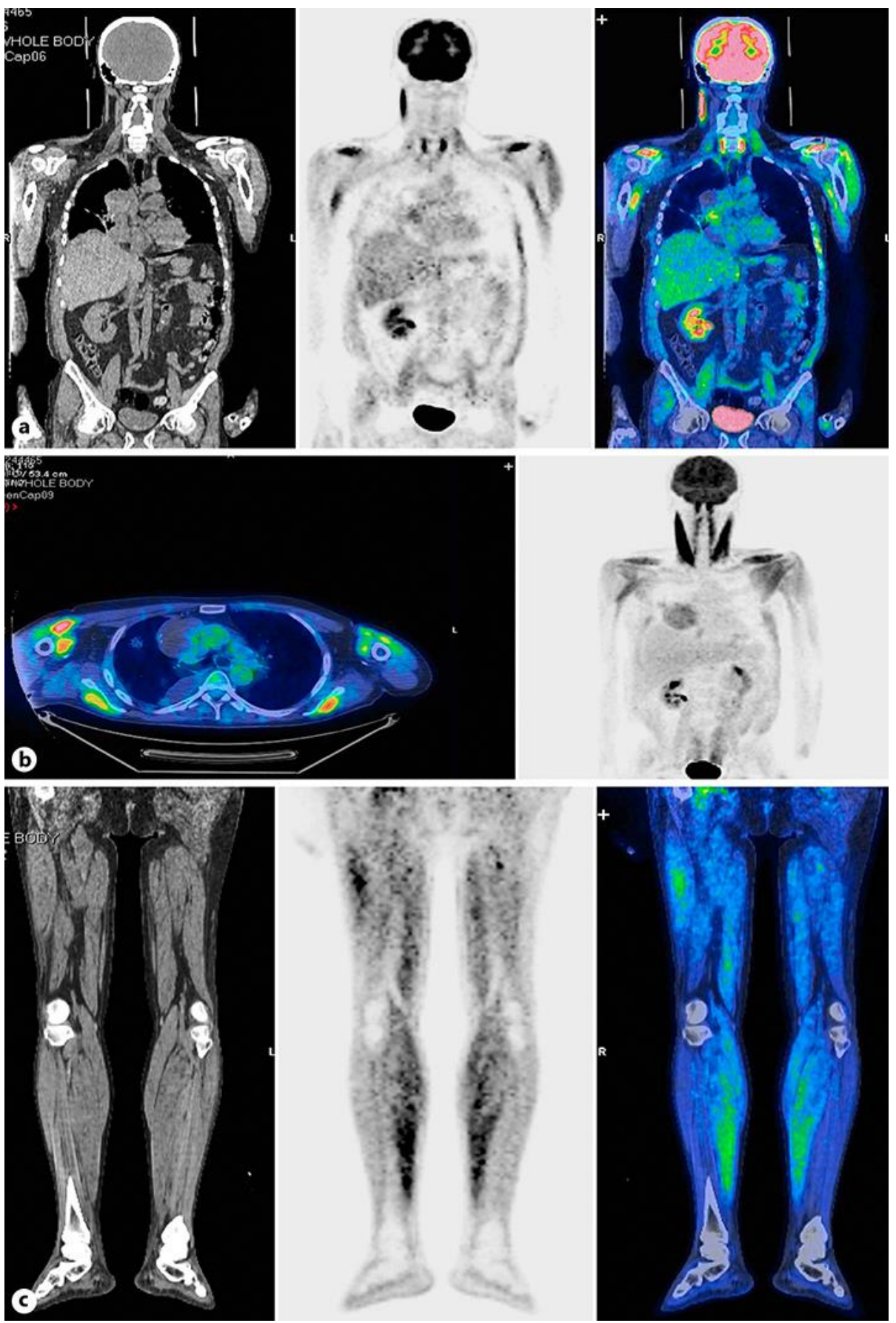

Fig. 1. Anteroposterior maximum intensity projection ${ }^{18}$ F-FDG positron emission tomography image showing intense abnormal tracer uptake into the skeletal muscles, in particular, into the muscles of the upper extremities, the long muscles of the neck, muscles of the chest wall, and sternocleidomastoid muscle (a, b) as well as the muscle groups of the lower limbs (c). 


\section{Case Reports in Oncology}

\begin{tabular}{l|l} 
Case Rep Oncol 2017;10:258-264 \\
\hline DOI: 10.1159/000464440 & $\begin{array}{l}\text { (c) 2017 The Author(s). Published by S. Karger AG, Basel } \\
\text { www.karger.com/cro }\end{array}$ \\
\hline
\end{tabular}

Damato et al.: Severe Rhabdomyolysis during Treatment with Trabectedin in Combination with a Herbal Drug in a Patient with Metastatic Synovial Sarcoma

Table 1. Laboratory parameters related to rhabdomyolysis starting from the first day of hospitalization

\begin{tabular}{lcrrrrr}
\hline Laboratory test & Normal range & Day 1 & Day 5 & Day 9 & Day 17 & Day 24 \\
\hline Myoglobin, ng/ml & $0.00-110.0$ & $14,869.5$ & $2,511.1$ & 310.8 & 230.5 & 94.7 \\
Creatine phosphokinase, U/L & $32-294$ & 3,009 & 1,306 & 217 & 211 & 76 \\
Aspartate aminotransferase, U/L & $2.0-40.0$ & 428 & 214 & 62 & 40 & 23 \\
Alanine aminotransferase, U/L & $4.0-49.0$ & 497 & 330 & 177 & 61 & 31 \\
Alkaline phosphatase, U/L & $90-360$ & 756 & 803 & 578 & 454 & 333 \\
Gamma glutamyl transpeptidase, U/L & $5.0-73.0$ & 918 & 789 & 511 & 288 & 154 \\
Lactate dehydrogenase, U/L & $208-379$ & 1,640 & 1,751 & 1,312 & 1,197 & 804 \\
\hline
\end{tabular}

